Relations industrielles

Industrial Relations

\title{
Rebâtir la confiance après une grève de longue durée
} est-ce possible?

Reconstruir la confianza después de una huelga muy larga

es posible?

\section{Re-establishing Trust After a Long-Term Strike}

Is It Possible?

\section{Geneviève A. Bonin et Denis Harrisson}

Volume 63, numéro 2, 2008

URI : https://id.erudit.org/iderudit/018575ar

DOI : https://doi.org/10.7202/018575ar

Aller au sommaire du numéro

Éditeur(s)

Département des relations industrielles de l'Université Laval

ISSN

0034-379X (imprimé)

1703-8138 (numérique)

Découvrir la revue

\section{Citer cet article}

Bonin, G. A. \& Harrisson, D. (2008). Rebâtir la confiance après une grève de longue durée : est-ce possible ? Relations industrielles / Industrial Relations, 63(2), 246-267. https://doi.org/10.7202/018575ar
Résumé de l'article

Cette étude a pour but d'analyser les facteurs contribuant à bâtir la confiance entre employés syndiqués et cadres après une grève de longue durée dans une succursale bancaire. Des entrevues semi-dirigées auprès de treize employés et cinq cadres, des observations et une analyse documentaire ont servi à déterminer ces facteurs. Ces derniers ont été analysés à la lumière des écrits scientifiques pour établir un modèle relationnel et analyser la situation vécue par les membres de l'organisation à l'étude.
Tous droits réservés @ C Département des relations industrielles de l'Université Laval, 2008
Ce document est protégé par la loi sur le droit d'auteur. L'utilisation des services d'Érudit (y compris la reproduction) est assujettie à sa politique d'utilisation que vous pouvez consulter en ligne.

https://apropos.erudit.org/fr/usagers/politique-dutilisation/ 


\title{
Rebâtir la confiance après une grève de longue durée
}

\section{Est-ce possible?}

\author{
Geneviève A. Bonin \\ DeNIS HARRISSON
}

Cette étude a pour but d'analyser les facteurs contribuant à bâtir la confiance entre employés syndiqués et cadres après une grève de longue durée dans une succursale bancaire. Des entrevues semi-dirigées auprès de treize employés et cinq cadres, des observations et une analyse documentaire ont servi à déterminer ces facteurs. Ces derniers ont été analysés à la lumière des écrits scientifiques pour établir un modèle relationnel et analyser la situation vécue par les membres de l'organisation à l'étude.

Les travaux de recherche portant sur les effets des grèves intéressent les chercheurs et les praticiens en relations industrielles. Au Québec, si les statistiques montrent une réduction significative des grèves au cours des dernières années (Sauvé et Robitaille, 2005), l'intérêt n'en demeure pas moins présent, car les grèves de longue durée sont plus apparentes. Cette recherche porte sur la confiance entre cadres et employés syndiqués dans un contexte de retour au travail après une grève de plus d'une année. Nous cherchons à comprendre le phénomène de la confiance dans une situation de conflit organisationnel. Plus précisément, l'objectif de cette étude est d'analyser les facteurs qui contribuent à rétablir la confiance entre gestionnaires et salariés syndiqués à la suite d'un conflit majeur.

- Bonin, G., M.B.A., doctorante en communication, Université McGill, Département d'histoire de l'art et des études en communication, Montréal, Québec, genevieve.bonin@ mail.mcgill.ca

- Harrisson, D., Ph. D., professeur titulaire, Université du Québec à Montréal, École des Sciences de la Gestion, Département d'organisation et ressources humaines et Directeur du Centre de recherche sur les innovations sociales (CRISES), harrisson.denis@uqam.ca 
Il existe deux types de travaux de recherche consacrés aux grèves. Les premiers portent sur l'impact économique des grèves telles que les fluctuations des actions à la bourse (Kramer et Vasconcellos, 1996; Clark, 1996; McDonald et Bloch, 1996; Persons, 1995), les salaires (Stengos et Swindinsky, 1990), les analyses socio-économiques (Mehling, 1963), la croissance et la productivité des entreprises ayant subi un arrêt de travail (Naples, 1981, Knight, 1989) et la situation financière des familles (Radecki, 1981; Gennard, 1981). Les seconds informent sur les effets psychosociologiques des grèves, notamment les liens entre les familles et la communauté touchée par une grève, les liens entre employés et cadres au retour d'une grève (Radecki, 1981; Gennard, 1981).

Les études mentionnent l'importance de la confiance, la justice et la communication entre les agents du travail dans le contexte de retour au travail (Lewicki et Bunker, 1996; Ethier, 1991; Radecki, 1981), sans toutefois en faire l'objet central de l'étude. La confiance est essentielle à la solidarité, la coopération, la productivité, la performance de groupe, la satisfaction des besoins, la participation, le changement organisationnel, le contrat psychologique et la communication (Barber, 1983; Blau, 1964; Golembiewski et McConckie, 1975 ; Rotter, 1967, Zand, 1972; Boss, 1978, Deutsch, 1973 ; Loomis, 1959; Sherif, 1966; Maslow, 1954; Golembiewski, 1986; Miles et Ritchie, 1984; Mellinger, 1956). C'est pourquoi on y porte un intérêt croissant dans les recherches sur les organisations. Notre étude cherche à identifier et à analyser les facteurs qui contribuent à la construction de la confiance alors qu'aucun élément ne semble a priori y souscrire puisque les agents du travail se remettent d'un long et périlleux conflit qui n'a engendré que de la méfiance des uns envers les autres.

Pour réaliser la recherche, nous avons effectué une étude de cas auprès de la Banque des Quatre-saisons ${ }^{1}$. En raison des stigmates profonds sur les relations entre les parties qu'engendre tout conflit, il est très difficile de trouver un ou des cas désirant se prêter à l'objet de la recherche. Cette entreprise a accepté à la condition ultime de ne pas être identifiée. De 1995 à 2004, la durée moyenne des grèves de ce secteur d'activité était de cinquante-deux jours au Canada (RHDC, 2005), alors que les employés de cette banque ont été en grève pendant quatorze mois. Nous supportons

1. Bien que la situation décrite au cours de cette étude soit réelle, toutes les informations pouvant révéler l'identité de l'entreprise ou des employés ayant participé à l'étude, le secteur d'activité et la durée du conflit ont été omises afin de maintenir l'anonymat de l'entreprise et de ses employés. Ceci était aussi nécessaire pour respecter l'entente de confidentialité entre la direction de l'entreprise et les chercheurs. Par conséquent, la Banque des Quatre-saisons remplace le nom réel de l'entreprise tout au long de l'étude. Les références exactes aux articles de journaux dont il est question dans le cas ont aussi été omises par respect de cette entente. 
l'idée que la confiance peut être reconstruite à partir d'éléments structuraux et d'une longue et patiente reconstruction des relations entre les personnes. Voyons auparavant quelques aspects théoriques de la confiance.

\section{QUELQUES REPÈRES THÉORIQUES}

Le type de négociation qui favorise une structure de jeux où il $\mathrm{y}$ a un gagnant et un perdant produit des effets sur le retour au travail. Même après la signature d'une convention collective, des conflits interpersonnels peuvent perdurer et d'autres méthodes de résolutions de conflits doivent alors être utilisées, telles les interventions en développement organisationnel auprès de groupes de travail (Éthier, 1991), les relations fondées sur des objectifs (relationships by objectives) (Hebdon et Mazerolle, 1995) ou encore les méthodes de formation au travail d'équipe (team building) (Blake et Mouton, 1975). Ces techniques nécessitent toutefois un niveau de confiance assez important qui n'existe pas toujours lors d'un retour au travail. Avant de faire un choix, il est important de connaître le niveau de confiance qui existe entre les parties (Stephenson et Pops, 1989; Paquet, Gaétan et Bergeron, 2000).

La confiance entre gestionnaires et salariés repose sur la croyance que l'autre partie agira toujours de bonne foi dans ses actions et ses paroles, selon les règles et les normes établies de façon implicite ou explicite par la convention collective ou tout autre cadre référentiel de l'entreprise de manière à assurer une forme de coopération qui saura répondre aux attentes, aux besoins et aux intérêts mutuels (Giddens, 1990; Cummings et Bromiley, 1995; Harrisson et Laplante, 1994). La confiance n'est pas aveugle, elle coexiste avec la méfiance car, comme le soutiennent Lewicki, McAllister et Bies (1998), la tension dynamique entre la confiance et la méfiance est nécessaire pour faciliter les relations harmonieuses dans les organisations. La méfiance représente un comportement négatif, elle démontre un manque de confiance totale d'un membre syndiqué ou d'un gestionnaire qui aurait, dans ses paroles ou ses gestes, agi contre les règles et les normes établies des formes de communication verbale et non verbale.

Il existe plusieurs définitions de la confiance. Lewicki et Bunker (1996 : 119-122) propose trois niveaux de confiance d'une relation donnée. Le premier niveau réfère à la confiance basée sur le calcul, insinuant que les personnes se font confiance en calculant les gains produits par la réciprocité de la relation. Ce type de confiance est facile à obtenir, en corrolaire la relation est encore plus facile à briser dès qu'un des agents fait défection ou qu'il n'y trouve pas son compte dans la relation de réciprocité. C'est d'abord de cette façon que se construit la confiance entre personnes qui ne se connaissent pas et qui désirent établir une relation de coopération. Le 
deuxième niveau de confiance est basé sur la connaissance : des personnes se connaissent suffisamment bien qu'elles peuvent construire une relation par échanges réciproques d'informations, établissant ainsi leur réputation. La relation de confiance s'inscrit alors dans une certaine pérennité. $\mathrm{Ce}$ type de relations de confiance est recherché par les personnes qui veulent établir des relations durables. Enfin, le troisième niveau est atteint lorsque les personnes appartenant à un groupe ou une catégorie sociale spécifique partagent des traits identitaires qui facilitent les liens de confiance. Dans les organisations, la confiance repose le plus souvent sur le calcul. Elle peut atteindre le stade de la confiance de type cognitif seulement si les acteurs font des efforts en ce sens en créant des dispositifs de confiance permettant l'échange d'informations, parfois en fonction de certains buts à atteindre. La confiance identitaire est le propre des groupes homogènes et elle est plutôt rare dans les organisations sinon dans les groupes de travail ou dans certaines catégories professionnelles (Harrisson, 2003).

Cette catégorisation de la confiance est produite à la suite de celle qu'avait établie Zucker (1986) : la confiance basée sur les relations d'échanges précédentes ou la réputation, la confiance liée aux caractéristiques de l'individu et à sa culture, puis la confiance construite selon des structures sociales et les institutions. Dans le cadre de notre étude, ce dernier type de confiance est intéressant, car il invite les agents du travail à se faire confiance par le biais d'une entente collective reconnue au plan institutionnel. Cependant, c'est nettement insuffisant si les agents recherchent davantage la coopération dans un contexte de grande incertitude. C'est pourquoi ils désirent également établir des liens de confiance construits par des échanges multiples. Plus il y a d'échanges, plus la confiance cognitive est élevée. Toutefois, l'entreprise n'est pas l'endroit privilégié pour avoir de tels échanges (Kramer, Brewer et Hanna, 1996). Certaines conditions comme l'identification à l'organisation et à ses membres permettent néanmoins d'accroître la confiance. La confiance peut également être motivée par la réciprocité, l'encouragement, la compensation et la morale. Au plan institutionnel, la confiance provient principalement des rôles, des règles et de la structure de l'organisation attribuables à la haute direction (McCauley et Khunert, 1992). Lorsque les employés perçoivent positivement la direction de l'entreprise, ils peuvent y accorder leur confiance. De plus, cette perception peut s'améliorer si, en gestion des ressources humaines, on fait la promotion de l'apprentissage, des possibilités d'avancement, de la stabilité et de l'équité (Costigan et al., 2004). La confiance est un phénomène de réciprocité propre à des relations entre des personnes qui représentent des intérêts encadrés au plan institutionnel, et la confiance est fortement influencée par des facteurs institutionnels et des conditions préalables. 
Cette étude repose sur une approche institutionnelle selon laquelle des formes ordonnées et intégrées émergent d'activités quotidiennes qui sont peu organisées ou instables (Broom et Selznick, 1955 : 238), comme le sont les relations propres à un retour au travail après une grève qui a laissé des séquelles sur la condition des personnes et leur volonté à structurer des relations établies sur la confiance. Les rencontres entre les agents supposent une structure formelle de laquelle émergent des normes informelles, des pratiques administratives et des connaissances qui résultent d'un historique commun dans la recherche d'un but, la résolution d'un problème ou l'adaptation à une situation donnée (Selznick, 1996 : 271). L'approche néo-institutionnelle rejette les modèles d'acteurs rationnels et l'intérêt porté envers les institutions en tant que variables indépendantes (Powell et Dimaggio, 1991). Le néo-institutionnalisme se veut plutôt une perspective qui se tourne vers les explications culturelles et cognitives, aux unités d'analyses « supra-individuelles » qui ne peuvent être réduites à des groupes agrégés ou encore à des motifs ou des conséquences directes des attributs individuels (Powell et Dimaggio, 1991 : 8). Dans les organisations, les membres partagent des valeurs, des façons de concevoir la réalité mais, dans certains cas, ils entrent en conflit sur le sens à donner à cet univers partagé, car ils possèdent des connaissances qui les autorisent à interpréter ce qui se produit dans leur organisation. Concrètement, ils interviennent dans des sous-systèmes qui sont aussi influencés par plusieurs autres institutions, dont les médias, l'État et le système de relations industrielles par exemple. Les concepts de confiance et de méfiance, sujets de cette étude, découlent de ces institutions qui révèlent l'importance d'une perspective de recherche qui tient compte de ces multiples dimensions. Compte tenu du contexte, les résultats sont à la fois des constructions sociales des participants et des influences institutionnelles omniprésentes dans les organisations contemporaines (Zucker, 1986; Berger et Luckmann, 1989; Powell et Dimaggio, 1991; Harrisson et Laplante, 1994). La prochaine section sert à détailler la méthodologie qui est issue de cette perspective théorique.

\section{LA MÉTHODOLOGIE}

L'étude a été réalisée six mois après le retour au travail en 2005. Treize salariés syndiqués (caissiers, conseillers, réceptionnistes et gardiens de sécurité) et cinq cadres (directeurs) ont été rencontrés pour un entretien de recherche, sur un total de quarante-six employés. Afin de minimiser l'impact de l'intervention sur le lieu de travail et pour éviter la répétition, des échantillons ont été choisis au hasard à l'intérieur des catégories et uniquement parmi les employés qui étaient présents au moment de la grève. Pour chaque catégorie où il y avait un nombre impair d'employés, 
cinquante pour cent des employés plus un ont été choisis, pour les catégories paires d'emplois, cinquante pour cent des employés ont été choisis et, finalement, cent pour cent des supérieurs ont été choisis compte tenu de leur petit nombre. Un seul employé a refusé d'être interviewé et les employés qui ne travaillaient pas à la banque au moment de la grève n'ont pas été sélectionnés.

Les informations recueillies reposent sur trois méthodes classiques de l'étude de cas : les entretiens semi-dirigés, l'observation non participante et l'analyse documentaire. Les entretiens enregistrés et retranscrits ont été d'une durée d'une heure et ils ont eu lieu six mois après la grève. Ils ont porté sur les relations entre les personnes, le climat de travail, le déroulement de la grève, le retour au travail, les facteurs contribuant à rétablir la confiance, le bris de confiance ainsi que sur les tâches et le travail en général. Seuls les entretiens avec le directeur général et le président du syndicat ont été plus spécifiques et exhaustifs, notamment sur le déroulement de la grève. Les entretiens ont eu lieu sur les lieux de travail dans un local prévu à cet effet.

L'observation a servi à analyser les comportements des personnes dans l'établissement. Les résultats de cet exercice ont permis d'assurer la cohérence entre le discours du participant et la situation réelle vécue par l'ensemble des employés. Ces observations sont limitées par les perceptions du chercheur. L'observation a porté sur les aspects suivants : aspect des lieux physiques et comportements entre les individus lors de la période d'entrevue.

L'analyse documentaire a porté sur les événements qui ont mené à la grève et l'information factuelle sur l'état des relations entre les individus avant, pendant et après le conflit. D'abord, les documents internes ont été consultés : journaux de grève, griefs, conventions collectives, dossier des incidents, déclarations solennelles, comptes rendus de négociation, lettres et mémos, ainsi que tout autre document pertinent ayant servi à relater les faits entourant la grève de la façon la plus juste et réaliste possible. Ces documents ont été fournis par les dirigeants de l'entreprise et les responsables syndicaux. Des articles de journaux ont aussi été consultés pour obtenir une vision externe du conflit. Ces articles provenaient principalement de cinq sources incluant trois grands quotidiens, sept journaux hebdomadaires, Radio-Canada en ligne et une revue diocésaine. Ils ont servi à décrire le cas et à corroborer les informations fournies par les personnes interrogées.

L'analyse a été effectuée selon les principes de la « théorie enracinée » de Glaser et Strauss (1967) afin de coder l'information de façon inductive. Les transcriptions d'entrevues et les autres documents écrits ont été codés selon les thèmes qui émergeaient des mots et expressions employés. Ces 
codes ont ensuite été classés et comparés à l'ensemble pour noter les similarités et disparités, en plus de certaines caractéristiques particulières. La triangulation des données a assuré la validité interne de la codification. Après avoir scruté les catégories à plusieurs reprises pour valider les thèmes récurrents, la théorie émergente a permis de créer des liens entre les catégories. Les liens et la théorie forment un modèle conceptuel qui répond à la question de recherche, soit celle de déterminer les éléments qui contribuent à construire la confiance. Le modèle comprend uniquement les informations qui répondent à cette question, éliminant ainsi toutes données sur la vie personnelle des gens, les anecdotes et les informations non liées au sujet de recherche.

\section{PRÉSENTATION DU CAS À L'ÉTUDE}

La Banque des Quatre-saisons est une entreprise privée opérant une dizaine de succursales à travers la province. Au total, plus de 300 employés assurent des services comparables à ceux des grandes banques à charte du pays. La succursale « $\mathrm{C}$ » est l'une des plus grandes succursales de la région comptant quarante-six employés (trois gardiens de sécurité, six caissiers juniors, deux caissiers aux comptes commerciaux, cinq conseillers financiers, trois réceptionnistes, trois caissiers seniors, trois caissiers aux comptoirs, cinq cadres (non syndiqués) et seize autres employés non syndiqués (agents de marketing, concierges, commis). Les employés syndiqués sont représentés par le syndicat des employés de la banque, affilié à une grande centrale syndicale canadienne. Au cours des quinze dernières années, cette succursale a connu trois grèves, la dernière étant la plus longue, soit quatorze mois. En plus de ternir l'image publique de l'entreprise, la grève a entraîné plusieurs incidents, de fortes tensions et des relations interpersonnelles plus tendues que jamais.

De nombreux délais ont ralenti les négociations à l'approche de la fin de la convention collective, incluant 191 jours sans négociation dus à l'absence du porte-parole syndical. La partie patronale propose une augmentation salariale de quatorze pour cent sur quatre ans, dont deux pour cent versés à un fonds de retraite, et un plancher d'emploi qui protège davantage de catégories d'emplois, passant de soixante-quinze à quatre-vingt-dix pour cent si le syndicat accepte la proposition salariale. Selon un journal local, le syndicat demande des augmentations salariales beaucoup plus élevées, un « véritable » fonds de retraite (à être négocié séparément de l'augmentation salariale), un plancher d'emploi incluant de nouveaux postes de conseillers financiers et de caissiers au comptoir et le changement de statut d'un poste de caissier au comptoir dont l'employé est surnuméraire, malgré vingt ans de service. Les offres patronales sont refusées à quatre-vingt-quinze pour 
cent par les membres de l'assemblée syndicale et la grève est déclenchée dès le lendemain.

Après un mois de grève, le syndicat reproche le désengagement progressif de l'entreprise envers la région et l'utilisation de briseurs de grève. Quoique légal pour ce type d'entreprise ${ }^{2}$, la partie patronale nie en avoir embauché. Ce sujet est au cœur des récriminations des grévistes durant toute la grève. À plusieurs reprises, les grévistes font appel à des intermédiaires pour en faire des alliés : journalistes, commerçants, clergé et élus politiques au niveau municipal, provincial et fédéral. Des représentations sont également faites auprès des clients afin de les inciter à boycotter la banque durant la durée de la grève. Les grévistes veulent démontrer des erreurs préjudiciables aux clients. Parmi les alliés potentiels, ces derniers comptent parmi les plus importants et influents. Ils sont incités à ne pas traverser les piquets de grève (journal local). De son côté, la partie patronale dénonce dans les journaux locaux les allocations non imposables des grévistes qui s'élevaient à 390 dollars par semaine après un mois de grève. Les grévistes utilisent comme mesure de représailles une pétition contre les services «pitoyables » de la banque avec l'intention de la faire parvenir à la direction juridique du ministère des Finances du Canada. Une plainte, de leur part, est également déposée au Conseil canadien des relations industrielles accusant l'entreprise de pratique déloyale et de perpétuer le conflit ${ }^{3}$.

Après huit mois, la grève perdure et de nouvelles lettres sont publiées dans les journaux envenimant davantage les relations et éliminant le peu de confiance qui existait. Les syndiqués reçoivent alors 450 dollars par semaine non imposable. Pour certains employés, cela représente plus que leurs salaires réels. L'entreprise est aussi la cible d'actes de vandalisme. Avec le temps, les manifestations jusque-là pacifiques dégénèrent pour faire place à des gestes plus durs : déboulonnement de roues de voiture, endommagement de biens de l'entreprise et des employés, déversement de fumier liquide sur une voiture, œufs, glace et matières fécales lancés tant sur les propriétés de l'entreprise que sur les propriétés privées des cadres. Malgré tout, les parties poursuivent les négociations.

La rancœur s'installe. Deux médiateurs et un conciliateur tentent de rapprocher les parties. De multiples rencontres, des manifestations et le temps ont raison de la grève. Finalement, après quatorze mois, une entente est acceptée à quatre-vingt-seize pour cent par les membres du syndicat. Elle inclut, entre autres, une augmentation salariale de neuf pour cent à la signature et de trois pour cent pour les années subséquentes pour un total de vingt-trois

2. Le Code canadien du travail (L.R.C. 1985, c. L-2) s'applique dans le présent cas.

3. Art. 94, Code canadien du travail. La décision n'est pas rendue durant la grève et, après le conflit, le Conseil décide de ne pas trancher (journal local). 
pour cent sur quatre ans et la création d'une caisse de retraite. Le plancher d'emploi est réduit, trois postes sont supprimés et d'autres sont fusionnés.

Les poursuites judiciaires sont abandonnées et les deux parties s'entendent sur un protocole de retour au travail qui est plus ou moins respecté. Il est toutefois difficile de croire que le retour au travail soit si pacifique compte tenu des interventions fréquentes de la police, l'usage d'appareils de surveillance de la part de la partie patronale, des habitudes de travail modifiées, de la perte de clientèle, des pertes financières et de la fatigue émotionnelle causée par cet événement.

\section{COMMENT RECONSTRUIRE LA CONFIANCE?}

L'analyse du discours des employés syndiqués et des cadres montre que les relations étaient « bonnes » avant la grève. Pendant la grève, elles se sont détériorées, devenant «mauvaises ». Comme le mentionne cet employé syndiqué : «J'essayais de pas être proche de la bâtisse, le moins possible pour euh... justement pour pas avoir à les [cadres] saluer le matin quand ils passaient $»^{4}$. Ou cette autre citation provenant aussi d'un employé : «Pendant le conflit, ça s'est largement détérioré. Ça c'est comme par exemple, une pyramide d'Égypte là, au fil des millénaires, veut veut pas la structure devient fragile, et même si elle est toujours en place là, y'a énormément de failles, et pendant le conflit, ces failles-là sont apparues parce que le conflit a été trop long », en référence aux problèmes latents au sein de la banque (problèmes de communication, différences de perspectives quant à la mission de l'organisation, etc.) qui ont été mis en évidence lors de la grève.

Après la grève, les employés disent avoir de l'espoir, mais selon plusieurs personnes interviewées, il y aura toujours de la méfiance. Un employé syndiqué mentionne : «... tous les autres autour là, mais je les vois comme... comme des rats. Le bateau en est infesté ». Les autres ont un niveau de confiance de base, c'est-à-dire de calcul, selon la typologie de Lewicki et Bunker (1996). Les personnes rencontrées ont trouvé des solutions pour rétablir la confiance. Celles-ci sont montrées dans les tableaux 1 et 2 . Le tableau 1 représente les solutions proposées par les employés syndiqués. La colonne de gauche comprend les facteurs de construction de la confiance identifiés par le chercheur, selon les codes employés lors de l'analyse des entrevues. La colonne de droite illustre quelques exemples de citations permettant de dégager les facteurs exposés dans la colonne de gauche. Pour des raisons d'espace, toutes les citations représentant les

4. Toutes les citations seront présentées au masculin pour assurer la confidentialité des sujets. De plus, toutes les données pouvant faire en sorte qu'on puisse reconnaître l'entreprise ou enfreindre l'entente de confidentialité seront remplacées par des données équivalentes dans le contexte de la banque. 
facteurs n'ont pas été exposées. Celles qui figurent dans le tableau ont été sélectionnées aléatoirement par le chercheur à titre d'exemple. Le code correspondant à la citation est identifié entre parenthèses. Le tableau 2 représente les solutions proposées par les cadres. Il a été élaboré de la même façon que le tableau 1.

\section{TABLEAU 1}

Moyens pouvant contribuer à augmenter la confiance entre employés syndiqués et cadres : perspective des employés syndiqués

Facteurs soulevés par les employés syndiqués

- Volonté

- Investissements matériels

- Encadrement

- Reconnaissance/ appréciation

- Communication

- Respect de la convention collective

- Formation des cadres

- Investissements humains

- Éviter la sous-traitance
Citations représentatives

(à titre d'exemple seulement)

«Ça prend de la volonté qu'on avance pour pas que le monde se sente qu'il y a pas de gagnants, puis y a pas de perdants » (volonté)

« Je pense qu'une bonne tape dans le dos ou une bonne reconnaissance en disant : "tu as fait du bon travail, félicitations" ce sont des choses qu'on a jamais-là... honnêtement c'est jamais dit : "tu as fait du beau travail, c'est beau" » (reconnaissance)

«Pour ce qui est de la communication, chez nous on sait que ça marche pas bien parce qu'il y a des informations qui ne se rendent pas aux autres là » (communication)

\section{TABLEAU 2}

Moyens pouvant contribuer à augmenter la confiance entre employés syndiqués et cadres : perspective des cadres
Facteurs soulevés par les
Citations représentatives
employés syndiqués
(à titre d'exemple seulement)

- Responsabilité

- Compréhension

- Communication

- Investissements matériels

- Vision d'entreprise

- Investissements humains

- Fournir des projets
« Le point qu'on a peut-être moins discuté, c'est l'effort que ç'a pris aux gens pour maintenir l'entreprise à flot. Je ne suis pas certain, puis je ne suis pas persuadé que les employés en grève comprennent jusqu'où ces employés-là ont fait des efforts pour maintenir l'entreprise » (compréhension)

«On ne communique pas assez. L'Internet là, c'est la bête noire. On ne se parle pas. On lit notre ordinateur, on ne se parle pas » (communication)

«Il faut avoir une vision d'entreprise pour amener ces gens à participer, à aimer l'entreprise, puis faut qu'ils aiment leur travail. Quand t'as une vision, t'as des objectifs, puis t'es partages. Y'en a pas là » (vision d'entreprise) 
Dans la section suivante, nous allons discuter de l'importance des facteurs présentés dans les tableaux, de la situation vécue par l'ensemble des employés et des cadres et ceci en lien avec les connaissances scientifiques.

\section{Analyse des facteurs communs soulevés}

Trois facteurs communs ont été soulevés par les cadres et les employés syndiqués. Ils sont : la communication, les investissements matériels et les investissements humains. Les deux parties estiment que la communication est déficiente sur plusieurs plans et ce serait l'obstacle principal au rétablissement de la confiance. D'abord, Internet est trop utilisé au détriment des rencontres en face à face diminuant ainsi la fréquence des échanges, mais aussi leur qualité. Des visites plus fréquentes des cadres qui ne sont pas toujours présents à la succursale seraient appréciées. Les repas sont souvent pris au poste du travail ou à l'extérieur plutôt que dans la salle commune. Rares sont les personnes qui se saluent dans les corridors. Il y a peu de sourires, de blagues ou de camaraderie familière. Les informations communiquées par les cadres sont aussi limitées aux discussions liées à la tâche plutôt que sur les informations financières que les employés aimeraient connaître ou encore des aspects stratégiques.

Plusieurs recherches ont souligné l'importance de la communication et des échanges d'information pour établir des relations saines (Koeszegi, 2004; Gillespie et Mann, 2004; Lewicki et Bunker, 1996; Roberts et O'Reilly, 1974), et rebâtir la confiance cognitive. Le simple fait de communiquer n'est pas suffisant. Encore faut-il qu'il y ait une qualité de la communication. Ce que les deux parties réclament, c'est « un dialogue vrai et honnête ». Ceci doit se faire fréquemment en face à face pour permettre la discussion et l'échange d'information au sujet des besoins, désirs et perspectives de résolutions de problèmes (Lewicki et Bunker, 1996). Ces échanges amélioreront la qualité de la relation (Koeszegi, 2004) et répondront au besoin privilégié des cadres et des employés (Harrisson, 2003). À la fréquence, s'ajoutent aussi le message authentique et sans déformation (Mishra, 1996), la perception de la satisfaction de la communication (Clampitt et Downs, 1993) et le contenu des informations. Les employés réclament davantage de documents écrits sur la situation financière pour renforcer les propos des dirigeants qui ne sont pas crédibles à leurs yeux. Ce manque de confiance peut-être attribuable aux difficultés récentes (Doost, 2003), au manque de leadership des dirigeants (Beslin et Reddin, 2004; Costigan et al., 2004) et au manque de circulation d'information quant aux valeurs communes de l'entreprise (Gillespie et Mann, 2004).

Les investissements dans le matériel physique de l'entreprise et l'investissement dans les ressources humaines de l'entreprise ont aussi été 
soulevés comme facteurs pouvant améliorer la confiance. Ils sont garants de la pérennité de la relation puisque les investissements symbolisent l'engagement à long terme. Cette observation va à l'encontre des études qui démontrent que l'investissement technologique contribue à réduire le nombre de salariés et à augmenter le contrôle exercé sur les employés (Kipnis, 1996). Les investissements en personnel et le remplacement des employés qui quittent ont aussi été identifiés par les cadres et les employés comme facteur essentiel à l'amélioration de la confiance. Mais comme le suggère un employé : « À moins qu'ils ne changent le staff au complet. Ils pourraient changer et faire en sorte que leurs paroles vont avec leurs actes, mais il faudrait que je le vois ». Les cadres jouent un rôle essentiel dans l'institution et si la confiance est brimée à cause d'eux, il sera impossible de rebâtir la confiance. Certains cadres, dont le directeur général de la banque, sont toujours dans l'organisation après trois grèves. Il est alors difficile de donner sa confiance, car ces agents représentent un univers que l'on veut révolu et la situation sociale semble alors irréversible.

\section{Analyse des facteurs soulevés par les employés syndiqués}

Les employés syndiqués ont noté six facteurs additionnels améliorant davantage leurs relations avec les cadres. La majorité des employés ont plusieurs années d'ancienneté, il y a donc déjà une prédisposition positive à faire confiance. Selon Taylor (2003), les personnes ayant des antécédents similaires encourageraient la volonté à se faire confiance. Aussi, une personne qui pressentirait un certain bénéfice aurait tendance à déployer des efforts afin de restaurer la confiance (Brockner et Siegel, 1996). Il s'agit donc d'un facteur positif sur lequel il est réaliste de mettre des énergies pour tenter de rétablir la confiance.

L'encadrement des cadres supérieurs crée également de l'insatisfaction auprès des employés syndiqués. Les employés soutiennent que les supérieurs n'ont jamais d'évaluations formelles ni de formation. Leur rémunération, selon eux, repose sur les économies réalisées sur le budget. Ces lacunes représentent pour les syndiqués un indicateur du manque de volonté et le peu d'importance accordée à la qualité des relations. La quête de la compétence professionnelle des employés syndiqués en relation avec la confiance n'est pas nouvelle (Harrisson, 2003), mais elle est un prédicteur de la confiance des employés (Costigan et al., 2004). Il y a aussi un encouragement pour un encadrement plus serré des employés en situation de confiance affaiblie et celui-ci doit être maintenu même dans un contexte de confiance où la tendance est de réduire considérablement l'encadrement ou la surveillance (Lee, 2004). La formation est perçue par les salariés comme un indicateur de l'engagement de l'entreprise envers eux (McCauley et Kuhnert, 1992; Gillespie et Mann, 2004), et donc une autre forme garante de la pérennité. 
« Je pense qu'une bonne tape dans le dos ou une bonne reconnaissance en disant : "tu as fait du bon travail, félicitations" ce sont des choses qu' on a jamais-là... honnêtement c'est jamais dit [...] », mentionne un employé syndiqué. Ce désir d'être reconnu et apprécié pour le travail bien fait est un besoin ressenti par tout employé spécialement les plus anciens qui n'ont pas connu cette gratitude depuis longtemps. Les récompenses et les mots d'encouragement augmentent la confiance des employés envers leurs supérieurs, toutefois, il doit déjà y avoir un minimum de confiance pour que l'employé prenne au sérieux les efforts du cadre pour souligner sa réussite (Gillespie et Mann, 2004 ; Costigan, Ilter et Burman, 1998). Un participant a d'ailleurs mentionné qu'il n'aurait pas cru un cadre s'il lui avait fait des éloges dès le retour de la grève, supposant ici une nouvelle disposition à être reconnu pour ses efforts qui démontre une certaine propension à oublier les événements du passé.

Le respect de la convention collective est normalement à la base de l'entente de retour au travail. Selon les employés syndiqués, les cadres devraient respecter leur choix de revendiquer un droit prescrit dans la convention collective. Ils affirment aussi que ce respect devrait s'étendre à l'acceptation de leur choix de faire la grève. Pour certains, les cadres ne respectent pas encore la décision qu'ils avaient prise. La convention collective est un engagement fortement institutionnalisé et comparable à une promesse qui, une fois brisée, est très difficile à rétablir. L'existence d'une convention collective suppose au préalable des relations basées sur la confiance (Harrisson et Laplante, 1994). Certains cadres n'avaient aucunement confiance au moment de la signature de la convention. Ceci expliquerait la facilité de ces derniers de ne pas, par exemple, suivre le protocole de retour au travail (un employé qui était censé être protégé par la convention n'a pas été rappelé lors du retour au travail). La manifestation du respect doit s'étendre à ce que Rodgers et Hunter (1991) décrivent comme étant la cohérence entre les paroles et les gestes des supérieurs. Le respect doit être mutuel et à la fois lié aux comportements des individus et à leurs traits personnels.

Le recours à la sous-traitance pour la réalisation de certains travaux est perçu par les employés syndiqués comme étant un manque de confiance de la part des supérieurs dans leurs capacités à fournir une prestation de qualité et à se fier à leur compétence. Durant la grève, les clients étaient souvent référés à des sous-traitants pour obtenir des services-conseils. Malgré le retour au travail, les agents de marketing, employés non syndiqués, font souvent la promotion des services-conseils externes. Les employés soutiennent que cette pratique va à l'encontre de la convention collective tout en les empêchant de mettre à profit leurs talents. Les études empiriques n'offrent pas d'indicateurs quant à la présence de ce facteur dans d'autres contextes. 


\section{Analyse des facteurs soulevés par les cadres}

Les facteurs qui sont uniquement soulevés par les cadres se limitent à quatre et comprennent : la prise de responsabilité, la compréhension, la vision d'entreprise et le développement de nouveaux projets. Plusieurs d'entre eux croient que la confiance s'accroît graduellement et que ce n'est que le temps qui améliorera les relations. Selon ces derniers, la prise de responsabilité va de pair avec la compréhension. Les employés syndiqués ne se rendent pas suffisamment compte des efforts fournis pour assurer la viabilité de l'entreprise en temps de grève. De plus, ils croient que les employés syndiqués devraient porter la responsabilité pour la perte de clients, la perte de notoriété et de crédibilité de la banque, la perte de revenu de la banque ainsi que les pertes de temps et d'énergie pendant et à la suite de la grève. Les cadres ne sont donc pas encore prêts d'oublier, mais il serait illusoire de croire que les employés peuvent adhérer à ces arguments irréconciliables avec leur réalité. Les intérêts opposés entre cadres et employés rendent impossible le partage de ce type de représentation qui ne tolère aucunement la reconnaissance du droit à la syndicalisation, la revendication et à l'exercice du droit de grève (Hebdon et Mazerolle, 1995). Par ailleurs, la confiance se base sur les attentes passées et des attentes anticipées (Zucker, 1986). Comme ce n'est pas le premier conflit dans l'entreprise et que ce n'est pas la première fois que les employés se sentent dupés dans leur relation avec leurs supérieurs, il est impossible pour eux de considérer une part de responsabilité dans ce contexte. Les cadres de l'entreprise sont identifiés comme étant les grands responsables. Il n'y a pas de démonstration à l'effet que l'on veuille construire sur de nouvelles bases.

L'absence de vision d'entreprise est un autre facteur mentionné par les cadres. Selon eux, il y a une vision globale pour l'entreprise, mais il n'y a pas de vision pour la succursale en question. Le directeur général est responsable de fixer un objectif particulier pour « son » établissement, lequel pourrait se greffer à la vision globale. Il doit également s'assurer que son message est transmis dans les divers services par les directeurs. Ce sont les valeurs, les objectifs et les décisions partagés qui réduisent l'incertitude au sujet du comportement futur des dirigeants et des salariés. Le partage de ces indicateurs rend difficile le bris de confiance (Gillespie et Mann, 2004). Une vision transmise et comprise efficacement par l'ensemble des employés est un aspect clé pour les relations de confiance. Les effets positifs d'une telle vision peuvent même encourager une tendance à vouloir partager les valeurs et la vision (Jones et George, 1998).

Finalement, les cadres estiment qu'en incitant les salariés à s'engager dans des projets structurants et motivants, ils se sentiraient davantage valorisés et développeraient une plus grande confiance en eux. Il se pourrait aussi que ces projets puissent être en lien avec la vision d'entreprise 
pour accroître davantage la confiance mutuelle des deux parties. Ceci est important surtout pour encourager et motiver les employés plus âgés (Duran et Klein, 1992).

\section{La relation entre les facteurs}

L'ensemble des facteurs dont nous venons de discuter ont permis, selon les indications des écrits scientifiques, d'établir des relations qui permettent d'exposer à la fois les demandes des employés syndiqués et des cadres en matière de confiance pour tenter de rétablir une relation durable. La figure 1 montre les liens et une hiérarchie possible ainsi que les facteurs qui incombent principalement à l'une ou l'autre des parties. La colonne de gauche démontre les facteurs émanant des employés syndiqués qui aideront à l'amélioration de la confiance. La colonne de droite représente les facteurs qui incombent aux cadres. Les pointillés dans cette colonne démontrent des facteurs et des liens non discutés dans la littérature scientifique, mais que nous croyons essentiels à la réussite du projet de confiance entre les deux parties. La colonne centrale montre les bienfaits des facteurs des autres colonnes, une fois mise en pratique. Certains facteurs comme les facteurs « temps » et « ambiance » ont été ajoutés parce qu'ils ont été soulevés à la fois dans la littérature ou dans les discours de façon indirecte.

FIGURE 1

Modèle des facteurs qui aident à l'amélioration de la confiance entre employés syndiqués et cadres

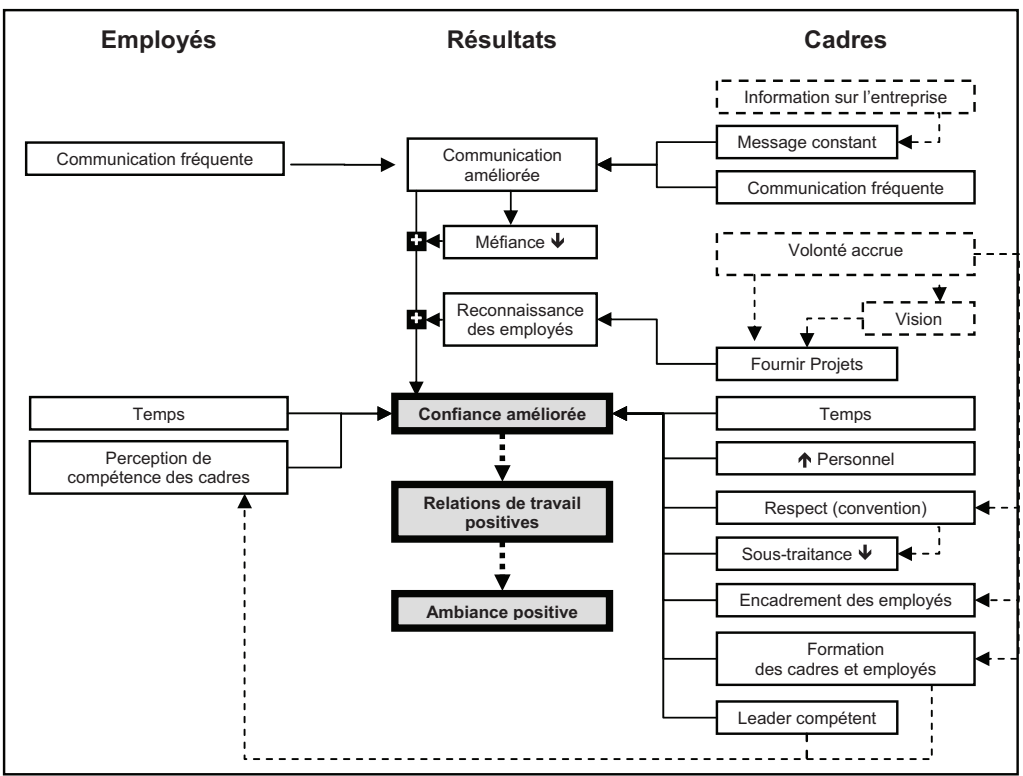


Bien que le modèle représenté par la figure 1 résume bien l'étude, il est évident que seules des études précises sur la valeur des facteurs et leur poids dans une relation de confiance d'après-grève pourraient assurer sa validité. Le rétablissement de la confiance après une grève de longue durée repose principalement sur les changements à l'échelle de l'entreprise, une sousinstitution affectée par des construits d'ordre à la fois social et culturel. Tant et aussi longtemps que les cadres tiennent à ce que les employés prennent la responsabilité pour la grève et ses conséquences, rien ne changera. Les employés n'oublient pas les bris de contrat, les promesses rompues et les contrevérités des supérieurs. Comme le mentionnent Lewicki et Bunker (1996), le facteur temps aidera à améliorer la situation si des changements dans la relation sont réalisés. Toutefois, des investissements en personnel, comme le suggèrent les deux parties, seraient idéaux pour stimuler de nouvelles relations saines. Des changements de personnel au niveau de certains postes clés ramèneraient une neutralité aux relations en diminuant les obstacles à la confiance que représentent les réminiscences du passé. Ce n'est pas une garantie, mais une indication en ce sens. Cela indique que l'on est prêt à effacer le passé. Les autres facteurs tels que la communication et la vision d'entreprise peuvent aussi être mis en commun auprès des deux parties, car l'un ne va pas sans l'autre. Une vision d'entreprise permettrait d'augmenter la crédibilité des cadres, car ils sauraient exactement où ils se dirigent et quels seraient les objectifs à atteindre. Comme l'ont souligné Hebdon et Mazerolle (1995), les relations par objectifs peuvent apporter une certaine réconciliation des parties. Ainsi donc, la réalisation de projets communs est un autre facteur qui pourrait être bénéfique.

\section{CONCLUSION}

La faible confiance entre les acteurs présente une difficulté à établir la confiance envers les institutions. En effet, la direction de l'entreprise ne reconnaît guère la présence syndicale et son rôle. Elle cherche plutôt à les engager dans leurs propres objectifs sans tenir compte de leurs propres valeurs et intérêts. C'est le sens de la grève de longue durée, l'entreprise ayant tenté de briser les liens syndicaux. Sans la reconnaissance de ce principe de base, il est difficile de faire confiance.

Cette étude visait à déterminer les facteurs contribuant à rebâtir la confiance entre cadres et employés syndiqués touchés par une grève de longue durée. D'un point de vue théorique, cette étude a permis d'identifier les lacunes en ce qui a trait aux relations bilatérales de confiance entre divers groupes d'employés, les effets de la grève sur le retour au travail, ainsi que la confiance dans un contexte d'après-grève. L'article fournit quelques pistes de recherches ultérieures. Premièrement, il serait intéressant de refaire cette 
étude dans une autre entreprise du même domaine ou encore d'un domaine différent ayant vécu une situation semblable. Deuxièmement, il serait bon de mesurer le poids des facteurs afin de savoir lesquels auraient le plus grand impact sur l'amélioration des relations entre cadres et employés syndiqués. Une troisième possibilité consisterait à mesurer le niveau réel de confiance afin de connaître l'ampleur du fossé qui s'est creusé entre les deux parties, faisant suite aux travaux menés par Tzafrir et Dolan (2004) sur la conception d'une échelle pour mesurer la confiance entre gestionnaires et employés. La présente recherche ouvre la porte à un sujet peu étudié qui a un impact important sur la vie des employés et des gestionnaires. Les études ultérieures devraient porter sur plusieurs cas et dans des secteurs variés.

\section{BIBLIOGRAPHIE}

BARBer, B. 1983. The Logic and Limits of Trust. New Brunswick, New Jersey : Rutgers University Press.

Berger, P. et T. Luckmann. 1989. The Social Construction of Reality : A Treatise in the Sociology of Knowledge. New York : Doubleday.

Beslin R. et C. REDDin. 2004. «How Leaders Can Communicate to Build Trust ». Ivey Business Journal Online, novembre/décembre, G1.

Blake, R. et J. Mouton. 1975. « Group and Organizational Team Building : A Theoretical Model for Intervening ». Theories of Group Processes. C.L. Cooper, dir. New York : Wiley, 103-129.

Blau, P.M. 1964. Exchange and Power in Social Life. New York : Wiley.

Boss, R.W. 1978. « Trust and Managerial Problem-Solving Revisited ». Group and Organization Studies, 3, 331-342.

Brockner, J. et P. SiEgel. 1996. "Understanding the Interaction between Procedural and Distributive Justice: The Role of Trust ». Trust in Organizations : Frontiers of Theory and Research. R.M. Kramer et T.R. Tyler, dir. Thousand Oaks, Calif. : Sage, 390-413.

Broom, L. et P. SElznick. 1955. Sociology : A Text with Adapted Readings. New York : Row, Peterson.

Clampitt, P. et C. Downs. 1993. «Employee Perceptions of the Relationship between Communication and Productivity : A Field Study ». The Journal of Business Communication, 30 (1), 5-28.

Clark, S. 1996. «Strike Behaviour when Market Share Matters ». Oxford Economic Papers, 48 (4), 618-639.

Costigan, R. et al. 2004. "Predictors of Employee Trust of their CEO : A Three-Country Study ». Journal of Management Issues, 16 (2), 197-216.

Costigan, R., S. Ilter et J. Burman. 1998. « A Multi-Dimensional Study of Trust in Organizations ». Journal of Managerial Issues, 10 (3), 303-317.

Cummings, L.L. et P. Bromiley. 1995. « The Organizational Trust Inventory (OTI) ». Trust in Organizations : Frontiers of Theory and Research. R.M. Kramer et T.R. Tyler, dir. Thousand Oaks, Calif. : Sage, 302-330. 
Deutsch, M. 1973. The Resolution of Conflict. New Haven, Connecticut : Yale University Press.

Doost, R. 2003. « Enron, Arthur Anderson and the Catholic Church : Are these Symptoms of a More Chronic Problem? ». Managerial Auditing Journal, 18 (1), 673-681.

DURAN, C. et B. KLEIN. 1992. « Managing Older Employees ». Leadership and Organization Development Journal, 13 (2), 1-2.

ETHIER, L.D. 1991. « The Effects on Intra- and Intergroup Team Building on Healing an Organization after a Labor Strike ». Thèse de doctorat, San Diego, California School of Professional Psychology.

GENNARD, J. 1981. « The Effects of Strike Activity on Households ». British Journal of Industrial Relations, 19 (3), 327-345.

Giddens, A. 1990. The Consequences of Modernity. Stanford : Stanford University Press.

Gillespie, N. et L. MANN. 2004. «Transformational Leadership and Shared Values : The Building Blocks of Trust ». Journal of Managerial Psychology, 19 (6), 588-607.

Glaser, B. et A. STRAuss. 1967. The Discovery of Grounded Theory : Strategies for Qualitative Research. Chicago : Aldine Publications.

Golembiewski, R. et M.L. MCCONCKIE. 1975. « The Centrality of Interpersonal Trust in Group Processes ». Theories of Group Processes. C.L. Cooper, dir. New York : Wiley, 131-185.

Golembiewski, R.T. 1986. «OD Perspective on High Performance : Some Good News and Some Bad News about Merit Pay». Review of Public Personnel Administration, 7, 9-27.

HARRISSON, D. 2003. «Les représentations de la confiance entre gestionnaires et représentants syndicaux : une analyse qualitative ». Relations industrielles/ Industrial Relations, 58 (1), 109-134.

HARRISSON, D. et N. LAPLANTE. 1994. « Confiance, coopération et partenariat ». Relations industrielles/Industrial Relations, 49 (4), 696-728.

Hebdon, R. et M. MAZEROlle. 1995. « Mending Fences, Building Bridges : The Effect of Relationship by Objectives on Conflict ». Relations industrielles/ Industrial Relations, 50 (1), 164-184.

HÉBERT, G. 1992. Traité de négociation collective. Boucherville : Gaëtan Morin éditeur.

Jones, G. et J. GeORGE. 1998. « The Experience and Evolution of Trust : Implications for Cooperation and Teamwork ». Academy of Management Review, 23 (3), 531-547.

KIPNIS, D. 1996. « Trust and Technology ». Trust in Organizations : Frontiers of Theory and Research. R.M. Kramer et T.R. Tyler, dir. Thousand Oaks, Calif. : Sage, 39-50.

KNight, K.G. 1989. "Labour Productivity and Strike Activity in British Manufacturing Industries : Some Quantitative Evidence ». British Journal of Industrial Relations, 27 (3), 365-375. 
Koeszegi, S. 2004. « Trust-Building Strategies in Inter-Organizational Negotiations ». Journal of Managerial Psychology, 19 (6), 640-660.

KRAMER, J.K. et G.M. VASCONCELLOS. 1996 « The Economic Effect of Strikes on the Shareholders of Nonstruck Competitors ». Industrial and Labor Relations Review, 49 (2), 213-227.

Kramer, R.M., M. Brewer et B. Hanna. 1996. «Collective Trust and Collective Action : The Decision to Trust as a Social Decision». Trust in Organizations : Frontiers of Theory and Research. R.M. Kramer et T.R. Tyler, dir. Thousand Oaks, Calif. : Sage, 357-389.

LEE, H. 2004. «The Role of Competence-Based Trust and Organizational Identification in Continuous Improvement ». Journal of Managerial Psychology, 19 (6), 623-629.

LEWICKI, R. et B. BUNKER. 1996. « Developing and Maintaining Trust in Work Relationships ». Trust in Organizations : Frontiers of Theory and Research. R.M. Kramer et T.R. Tyler, dir. Thousand Oaks, Calif. : Sage, 114-139.

Lewicki, R., D. MCAllister et R. Bies. 1998. «Trust and Distrust : New Relationships and Realities ». Academy of Management Review, 23 (3), 438-458.

Loomis, J. 1959. « Communication, the Development of Trust, and Cooperative Behavior ». Human Relations, 12, 305-315.

Maslow, A. 1954. Motivation and Personality. New York : Harper Collins.

MCCAuley, D. et K. KunNERT. 1992. «A Theoretical Review and Empirical Investigation of Employee Trust in Management ». Public Administration Quarterly, 16 (2), 265-284.

McDonald, J.T. et H. Bloch. 1996. « The Spillover Effects of Industrial Action on Firm Profitability ». Review of Industrial Organization, 15, 183-200.

Mehling, J. 1963. Analyse socio-économique d'une grève. Montréal : Les Presses de l'École des Hautes Études Commerciales.

MELLINGER, G.D. 1956. «Interpersonal Trust as a Factor in Communication ». Journal of Abnormal Social Psychology, 52, 304-309.

MiLES, R.E. et J.B. RitCHIE. 1984. « Participative Management : Quality versus Quantity ». Organizational Psychology : A Book of Reading. D.A. Kolb, I.A. Rubin et J.M. McIntyre, dir. Englewood Cliffs, New Jersey : PrenticeHall, 430-440.

Mishra, A. 1996. « The Centrality of Trust ». Trust in Organizations : Frontiers of Theory and Research. R.M. Kramer et T.R. Tyler, dir. Thousand Oaks, Calif. : Sage, 261-287.

NAPLeS, M.I. 1981. «Industrial Conflict and its Implications for Productivity Growth ». The American Economic Review, 71 (2), 36-41.

Paquet, R., I. GaÉtan et J.-G. Bergeron. 2000. « Does Interest-Based Bargaining (IBB) Really Make a Difference in Collective Bargaining Outcomes? ». Negotiation Journal, 16 (3), 281-296.

PERSONS, O.S. 1995. « The Effects of Automobile Strikes on the Stock Value of Steel Suppliers ». Industrial and Labor Relations Review, 49 (1), 78-87.

Powell, W. et P. DiMaggio. 1991. The New Institutionalism in Organizational Analysis. Chicago : The University of Chicago Press. 
RADECKI, H. 1979. The 1978-79 Strike at Inco: The Effects on Familie. A Report. Sudbury : Laurentian University Press.

RHDC (Ressources humaines et développement des compétences Canada, Direction des informations sur les milieux de travail). 2005. «Tableau et liste des arrêts de travail 1995-2004».

Roberts K.H. et C.A. O’ReILly. 1974. « Failure in Upward Communication : Three Possible Culprits ». Academy of Management Journal, 17 (2), 205-215.

RodGers, R. et J. Hunter. 1991. «Impact of Management by Objectives on Organizational Productivity ». Journal of Applied Psychology, 76 (2), 322-326.

RotTer, J. 1967. « A New Scale for the Measurement of Interpersonal Trust ». Journal of Personality, 35, 651-665.

Sauvé, M. et C. Robitaille. 2005. «Évolution des conflits de travail de longue durée de 1983 à 2002 : une vue d'ensemble ». Québec : ministère du Travail, Direction générale des politiques et de la recherche.

SELZNICK, P. 1996. "Institutionalism "Old" and "New" ». Administrative Science Quarterly, 41, 270-277.

SHERIF, M. 1966. In Common Predicament : Social Psychology of Intergroup Conflict and Cooperation. Boston : Houghton Mifflin.

Stengos, T. et R. Swindinsky. 1990. « The Wage Effects of the Strike: A Selectivity Bias Approach ». Applied Economics, 22 (3), 375-386.

Stephenson, M. et G. Pops. 1989. « Conflict Resolution Methods and the Policy Process ». Public Administration Review, 49 (5), 463-473.

TAYLOR, T. 2003. « Trust within Organisations ». Personnel Review, 32 (5), 556-568.

TZAFriR, S. et S. Dolan. 2004. « Trust Me : A Scale for Measuring ManagerEmployee Trust ». Management Research, 2 (2), 115-132.

ZAND, D.E. 1972. «Trust and Managerial Problem Solving ». Administrative Science Quarterly, 17, 229-239.

ZuCKER, L. 1986. «Production of Trust : Institutional Sources of Economic Structure, 1840-1920». Research in Organizational Behavior. B. Staw et L. Cummings, dir. Greenwich, Conn. : JAI Press, 53-111.

\section{SUMMARY}

\section{Re-establishing Trust After a Long-Term Strike: Is It Possible?}

The effects of strikes have always preoccupied industrial relations' scholars and practitioners. Even though statistically, there have been fewer strikes in Quebec during the last few years, they have not been of lesser interest. The most recent strikes have been more apparent because of their 
longer duration. This study discusses trust between managers and unionized employees who have returned to work after a fourteen-month strike. The objective is to better understand the phenomenon of trust in a situation of organizational conflict and, more precisely, to analyze the factors that contribute to re-establishing trust between union employees and managers following this major conflict.

A few theoretical concepts about trust help us understand the basic elements needed to produce collaborative relationships. Among other things, categories of trust established by Lewicki and Bunker (1996) as well as Zucker (1986) are presented to help make a better judgment with regard to the different types of relationships and to understand the way in which they present themselves in particular situations. This section ends with an overview of the authors' theoretical perspective, an institutional approach, which makes reference to Powell and Dimaggio's (1991) framework.

Because of the difficulty to access multiple companies affected by a strike, a qualitative case study was conducted. Thirteen union employees and five managers chosen randomly among each job category from a total of forty-six employees make up the sample. They were interviewed during semi-structured interviews six months following the end of the strike. Observations about individual behaviour during the interview period were also taken into consideration. An analysis of internal documents from the organization helped bring out factual information about the state of the relationship between the two groups before, during and after the strike. It is the use of these three traditional methods that helped construct the case presented. Not only does it explain the events surrounding the conflict by using a balanced approach including information obtained from union employees and company managers, but it also includes references to articles published in local newspapers to give a temporality and a sense of place to the events.

Subsequently, the authors undertake a discussion about ways to reconstruct trust among the parties. Tables detailing the factors that can contribute to reconstructing trust according to each party are presented with representative quotes from the semi-structured interviews. In total, three common factors were raised-communication, material investments and human investments. Communication is a central concept, but it also includes its share of complexities because the simple act of communicating is not sufficient. Even if, in basic terms, material investments are perceived as advantages, they are not in practice, according to the literature on this subject. Human investments can also bring new dynamics to a company, but cannot be done to the detrimental need of removing existing personnel. To these factors, the union employees add six additional ones including desire, supervision, recognition and appreciation, respect of the collective agreement 
and the importance of not resorting to outsourcing. The managers also came up with four additional factors-responsibility, comprehension, company vision and the importance of providing projects to union employees. All these factors are systematically analyzed in the article in direct relation to the written scientific and theoretical literature on these subjects.

In light of this analysis, it is possible to establish a relationship between these factors and a hierarchy of their importance that can serve to explore the possibility of reestablishing trust within the organization. These relationships and this hierarchy are presented in an illustration which summarizes the study. Explanations about its creation and the way to interpret it are also included. It is important, however, to note that although the study reveals a number of important factors needed to study trust, it has its limitations which can only be rectified by studying each factor in a distinct fashion and by weighing each one individually during subsequent research.

In conclusion, this research demonstrates the difficulty to establish trust within an organization because the company does not recognize the union's presence and its role. The company is rather searching to achieve its own objectives without taking the values and interests of its employees into account as well as those of the trade union. From a theoretical point of view, the study helps identify the limitations to trust relationships between various groups of employees, the effects of strikes on returning to work and of trust in the after-strike context. Paths for future research are explored. It is suggested to replicate the study in an institution in the same field or in different fields to open the door to the possibility of generalization. Secondly, it is suggested to weigh the factors to find out which ones would have the greatest impact on the creation of trust. And, finally, measuring the level of trust that really exists between the parties would be beneficial in understanding the scope of the division which separates them. 